\title{
Vascular Origins of BOLD and CBV $f$ MRI Signals: Statistical Mapping and Histological Sections Compared
}

\author{
Aneurin J Kennerley*, John E Mayhew, Peter Redgrave and Jason Berwick
}

\author{
Centre for Signal Processing in Neuroimaging and Systems Neuroscience (SPiNSN), Department of Psychology, \\ University of Sheffield, Western Bank, Sheffield S10 2TN, UK
}

\begin{abstract}
Comparison of 3T blood oxygenation level dependent (BOLD) and cerebral blood volume (CBV) activation maps to histological sections enables the spatial discrimination of functional magnetic resonance imaging (fMRI) signal changes into different vascular compartments. We use a standard gradient echo-echo planar imaging technique to measure BOLD signal changes in the somatosensory cortex in response to whisker stimulation. Corresponding changes in $\mathrm{CBV}$ were estimated following the infusion of a super-paramagnetic contrast agent. We imaged in a tangential imaging plane that covered the cortical surface. Images were associated with post mortem histological sections showing both the surface vasculature and cytochrome oxidase stained whisker barrel cortex. We found a significant BOLD signal change in the large draining veins which occurred in the absence of a corresponding CBV change. Results suggest that in the venous drainage system, $\sim 3 \mathrm{~mm}$ distant from the area of activity, there is a robust change in blood oxygen saturation with little or no volume change. CBV changes are localised over the somatosensory barrel cortex and overlying arterial supply, supporting the theory that CBV changes are greater in the arterial than in the venous vasculature. This work investigating BOLD signal and underlying hemodynamics provides more information on the vascular origins of these important neuroimaging signals.
\end{abstract}

Keywords: fMRI, BOLD, CBV, compartments.

\section{INTRODUCTION}

Functional magnetic resonance imaging ( $f \mathrm{MRI})$ is the brain imaging method at the forefront of contemporary cognitive neuroscience research. The most common $f$ MRI method, blood oxygenation level dependent (BOLD), relies on localised changes in blood oxygenation $[1,2]$ associated with neuronal activity. During neuronal activation there is an increase in cerebral blood flow (CBF) that outweighs increased oxygen consumption by 2:1 [3]. A 'washout' of the paramagnetic deoxyhemoglobin results and the tissue becomes hyperoxic. There is a dominant change in $\mathrm{R}_{2}{ }^{*}$ [4] measurable over time using, for example, a gradient echoecho planar imaging (GE-EPI) pulse sequence and this gives rise to a positive BOLD $f$ MRI signal. Accompanying these flow increases are localised increases in cerebral blood volume (CBV). Increased CBV will cause a net increase in $\mathrm{R}_{2}$ * counter-acting, but not overcoming, decreases in $\mathrm{R}_{2}$ * due to increased oxygenation. Mandeville et al. [5] utilised an intravascular paramagnetic contrast agent that maintained a steady state blood concentration in a rat model, to permit measurements of these CBV changes associated with neuronal activity.

Although the physical basis of the BOLD and CBV signals changes is well understood the biological and physiological bases (namely the underlying hemodynamics) of the $f$ MRI BOLD signal are less well appreciated. In particular, it is not clear how the different vascular

*Address correspondence to this author at the SPiNSN, University of Sheffield, Western Bank, Sheffield S10 2TN, UK; Tel: +44-114-222-6554; Fax: +44-114-276-6515; E-mail: A.J.Kennerley@shef.ac.uk compartments in brain tissue (arteries, capillaries and veins) contribute to the generation of the BOLD signal. The novelty of the current study is to investigate the spatial distribution and thus source of the BOLD and CBV $f$ MRI signal changes in a tangential plane parallel to the cortical surface. $f$ MRI statistical maps are compared to post mortem histological analysis to investigate the vascular origins of both the BOLD and cerebral blood volume (CBV) $f$ MRI signals.

The sources of stimulation-induced BOLD and CBV changes, in particular the spatial distributions of both the two signals have been extensively researched in rats [6-18], cats [19-21], and monkeys [22]. However, studies generally image in either the coronal plane $[6,16]$ or a similar plane perpendicular to the cortical surface [21]. This imaging plane is ideal for investigations into the depth profiles of the signals. Studies using gradient echo techniques show that the BOLD signal changes are greatest in magnitude at the cortical surface while CBV changes are often found in the deeper layers $[7,14]$. This has also been confirmed with spin echo techniques [6] and with investigation of sources of phase change [21]. Many attribute this difference to the fact that the BOLD signal is weighted towards the venous drainage system with coronal BOLD statistical maps often 'indicating' the presence of a large draining vein [6]. Indeed, venous BOLD signals have been demonstrated in both humans [23-25] and animals [6, 15], where, in one study responses from the vessels were greater than those from the tissue [26]. Venous BOLD signals could be a mechanical consequence of the elastic properties of the venous draining system. As CBF increases, the veins 'balloon' out (expand) producing transient mismatches between $\mathrm{CBV}$ and $\mathrm{CBF}[27$, 28]. Such a mechanism could result in a BOLD signal 
undershoot as the parameters return to baseline. The resulting changes in $\mathrm{CBV}$ and oxygenation in the venous vessels could give rise to a substantial BOLD signal in the draining veins.

However, several recent reports have also shown significant increases in $\mathrm{CBV}$ in the arteries. Optical microscopy studies revealed $\mathrm{CBV}$ changes in the arteries 5.3 times than those recorded from the venous compartment [29]. A study by Kim et al. [10] using arterial spin labelling (to measure $\mathrm{CBF}$ ) and an $\mathrm{MR}$ contrast agent (to measure $\mathrm{CBV}$ ) reported that the increased $\mathrm{CBV}$ during neural activation originated mainly from arterial rather than the venous compartments. They concluded that venous blood volume changes may be negligible in BOLD $f$ MRI. This conclusion is supported by vascular physiology studies showing that arterial vessels dilate by almost $20 \%$ during increased neural activity [30-32] while venous vessels maintain a constant diameter [31].

Uncertainties concerning the vascular origins of the BOLD signal and underlying hemodynamics indicate the area requires further investigation. Therefore, in the current study we measured both BOLD and CBV weighted $f$ MRI signal changes in the rat somatosensory cortex following whisker stimulation. Extending the previous investigation of Keilholz et al. [6], we measured $f$ MRI data from a tangential imaging plane parallel to the cortical surface (Fig. 1). This has the advantage over the often imaged coronal plane in that the entire structure of the vascular tree (arteries, capillaries and veins) can be investigated. We relate activated regions in $f$ MRI analysis to the vascular compartments revealed in the post mortem histological sections of barrel cortex. In particular, we concentrated on the $f$ MRI signal changes within a large vein draining the barrel cortex.

\section{METHODS}

\section{Surgery and Monitoring}

All aspects of these methods and their development were performed with UK Home Office approval under the Animals (Scientific Procedures) Act 1986. Female Hooded Lister rats $(n=6)$ weighing between $250 \mathrm{~g}$ and $400 \mathrm{~g}$ were kept in a 12-hour dark/light cycle environment at a temperature of $22^{\circ} \mathrm{C}$ with food and water ad libitum. Animals were anaesthetised with urethane $(1.25 \mathrm{~g} / \mathrm{kg}$ intra-peritoneal) and rectal temperature maintained at $37^{\circ} \mathrm{C}$ throughout using a homeothermic blanket (Harvard Apparatus). The left and right femoral veins and arteries were cannulated to allow drug infusion and measurement of mean arterial blood pressure, respectively. An RF surface coil (20mm diameter) was fixed to the animal's cranium with the somatosensory barrel cortex in the centre of the well. Whisker follicles are separately connected to the trigeminal nerve and the array of whiskers projects to the contra lateral somatosensory cortex. The whiskers, or sinus hairs are arranged in a set pattern with rows A (Dorsal regions) - E (Ventral regions). Each row is numbered in order in a caudal - rostral direction. To ensure the whole whisker pad and thus somatosensory barrel cortex was activated during electrical stimulation, two nonmagnetic electrodes were inserted into the left whisker pad, between rows $\mathrm{A} / \mathrm{B}$ and $\mathrm{D} / \mathrm{E}$ in a posterior direction.

The animal was secured in a magnet-compatible holding capsule [7]. The animal was artificially ventilated (parameters were adjusted for individual animals to maintain blood gas measurements of $\mathrm{pCO}_{2}$ in the range $32-40 \mathrm{~mm} \mathrm{Hg}$ ) and blood pressure monitored throughout.

\section{fMRI Techniques}

The capsule was placed into a 3 Tesla magnet (Magnex Scientific Ltd., MRRS console), fitted with a $10 \mathrm{~cm}$ inner diameter, self shielded and water cooled gradient set (Magnex Scientific Ltd. $10 \mathrm{kHz} / \mathrm{mm}$ maximum strength per axis with $100 \mu \mathrm{s}$ ramps). High spatial resolution gradient echo scans were performed $(256 * 256$ pixels, $\mathrm{FOV}=30 \mathrm{~mm}$, slice thickness $=2 \mathrm{~mm}, \mathrm{TR} / \mathrm{TE}=1000 / 15 \mathrm{~ms}$, flip angle $=90^{\circ}, 2$ averages). Using a standard coronal section (Fig. 1a) we identified an oblique slice to allow tangential imaging of the cortical surface vasculature (Fig. 1b).

BOLD functional data were acquired from the oblique slice using a modulus blipped echo planar single shot technique (MBEST) during electrical stimulation (raw data matrix $=32 * 64$ zero padded to $64 * 64$, data sampling interval $=5 \mu \mathrm{s}, \quad \mathrm{FOV}=30 \mathrm{~mm}$, slice thickness $=2 \mathrm{~mm}$, TR/TE=1000/15ms, flip angle $90^{\circ}, 2$ averages). Read-out direction for this Gradient Echo-Echo Planar Imaging (GE-

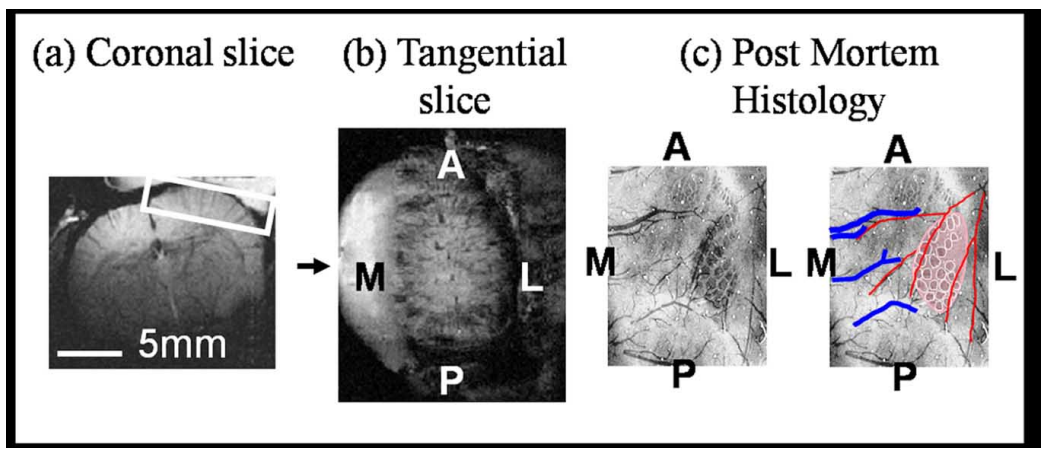

Fig. (1). Experimental data orientations. Geometry is given in the anatomical reference frame. One representative animal shown. a) Coronal structural MR image $\left(256^{*} 256\right)$ allows placement of b) a tangential slice covering the right hand cerebral hemisphere and hence surface of the somatosensory cortex (A-Anterior, P-Posterior, M-Medial and L-Lateral). BOLD and CBV activation maps superimposed onto such tangential sections were directly compared to post mortem histological sections (c). Photographic emulsion (previously injected into the vascular system) reveals the pattern of arteries (red) and veins (blue) in the somatosensory cortex. Individual barrels in the somatosensory cortex contain high levels of cytochrome oxidase. Staining for this enzyme allows direct visualisation of the whisker barrel field. 
EPI) sequence was left-right for both slice orientations. Standard phase correction [33] was used to minimise Nyquist ghosting.

Following completion of BOLD activation studies, we infused a Monocrystalline Iron Oxide Nano-particle (MION) super-paramagnetic contrast agent AMI-227 SINEREM (supplied by Guerbet, France) to estimate the changes in cerebral blood volume. The diameter of the particle is $30 \mathrm{~nm}$ much smaller than that of a red blood cell $(\sim 5 \mu \mathrm{m})$ and so circulated through the vascular system with ease. At a dose of $200 \mu \mathrm{mol} / \mathrm{kg}$ MION has a half life of 4.5 hours in the rat. We therefore infused a total dose of $160 \mu \mathrm{mol} / \mathrm{kg}$ via the femoral vein. At this dose the MION contrast agent would be expected to dominate the deoxyhemoglobin contribution to the blood magnetisation. Therefore, MR signal variations evoked by stimulation of the whisker pad under these conditions would reflect changes in cerebral blood volume and not BOLD signal [5].

\section{Stimulus Protocol}

Local hemodynamic changes in the somatosensory cortex were induced by electrical stimulation of the whisker pad (1.2mA, $5 \mathrm{~Hz}$ for $16 \mathrm{~s})$. A 780 second trial consisted of an initial 60 second control period followed by 7 blocks of $16 \mathrm{~s}$ stimulation separated by inter stimulus intervals of 96 seconds. This schedule allowed hemodynamic activity in the cortex to return to a baseline state before the next stimulus. The timing of stimulus delivery was controlled by a CED1401 locked to MR echo acquisition.

\section{Data Analysis}

Data analysis was conducted using Matlab (The MathWorks Inc.). After GE-EPI to GE structural image registration all $f$ MRI data were analysed using the general linear model (GLM) approach as in SPM [34]. The time series across each pixel was compared to a design matrix comprised of seven representative hemodynamic responses (box-car design), a ramp and a DC offset. Activation zscores were calculated on a voxel by voxel basis to identify active regions. A region was defined 'active' if at least five adjacent voxels each had z-scores greater than four. The individual design matrix components of any qualifying events were combined into a single vector. SPM analysis was then re-applied to check for statistical relevance to the 'combined' design matrix. Z-scores were superimposed on detailed structural MR scans to identify active regions. This 'optimised' approach corrects the statistical analysis for any stimulation trials that produce no response, which is thought to occur due to the cortical state of the animal; a potential by-product of anaesthesia. Time series of responses were taken as the mean across qualifying trials and subjects.

\section{Histology}

After experimental testing the animals were prepared for cytochrome oxidase histochemistry. First, photographic emulsion was injected into the vascular system to reveal the pattern of arteries and veins in somatosensory cortex. Individual barrels in rodent somatosensory cortex contain high concentrations of the enzyme cytochrome oxidase. Consequently, histological processing to reveal this enzyme results in a map of the whisker barrel field. Images of the vasculature were superimposed on those of sections stained for cytochrome oxidase (Fig. 1c). These maps were compared to statistical maps of $f$ MRI BOLD and CBV signals (Fig. 2). Details of this methodology have been reported previously [35].

\section{RESULTS}

Cortical activation during electrical stimulation of the whisker pad was observed with both BOLD and CBV weighted $f$ MRI in all rats. In both BOLD and CBV experiments, all animals tested showed a response localised over the contra-lateral barrel region (Fig. 2). Activation maps were compared directly to post mortem histological sections. In the BOLD images we found an area of activated pixels extending from the barrel cortex to the superior sagittal sinus. This region coincided with one of the large draining veins seen in the histology and thus supports previous studies in the coronal plane which were only indicative of a venous BOLD response $[6,15]$. Interestingly, the $\mathrm{CBV}$ weighted images show no corresponding changes in activity in the draining veins. In the CBV maps, activation showed significant confinement to the parenchymal regions of whisker somatosensory cortex and the overlying arterial supply.

To obtain time series, two regions of interest were selected. One covered the barrel cortex, and the second was centred on the draining vein region seen in the BOLD maps. The averaged time series of BOLD and CBV from both the barrel and venous regions are shown in Fig. (2c). The BOLD response in the barrel and venous regions had similar temporal dynamics with both responses peaking at 3.0-2.5\%. However, upon interpolation $(0.5 \mathrm{~Hz}$ to $20 \mathrm{~Hz})$ of the time series and normalisation between the pre-stimulus baseline and the response plateau (4 to $16 \mathrm{~s}$ after stimulus onset) of the two responses we found that the venous response lagged the barrel response by $0.55 \mathrm{~s} \pm 0.09$. This delay was calculated across animals as the time between the two responses as they reached a normalised magnitude of 0.5 on initial stimulus rise (0 to $4 \mathrm{~s})$. This delay was found to be significant using a 't-test' at the 5\% significance level. Note that neither time series provides evidence for a significant 'deoxy-dip' or post stimuli undershoot. Both returned to baseline 26-30s after the stimulus had ceased.

In contrast, the time series of the CBV response from the same barrel and venous regions had a very different temporal profile. A $20 \%$ change in cerebral blood volume was observed in the barrel brain parenchyma, but no change was detected in the region of the draining vein. This suggests the BOLD response seen in the large draining vein may have been caused solely by a decrease in deoxyhemoglobin in the absence of any blood volume change. Moreover, the duration of the CBV response in barrel cortex was longer, extending to approximately $46 \mathrm{~s}$ after stimulus off-set.

\section{DISCUSSION}

Our main finding was that hemodynamic responses reflected in BOLD and CBV-weighted $f \mathrm{MRI}$ data were different depending on the vascular compartment. When the functional imaging maps were related to corresponding histological sections of whisker barrel cortex and surrounding vasculature, the BOLD signal was weighted 


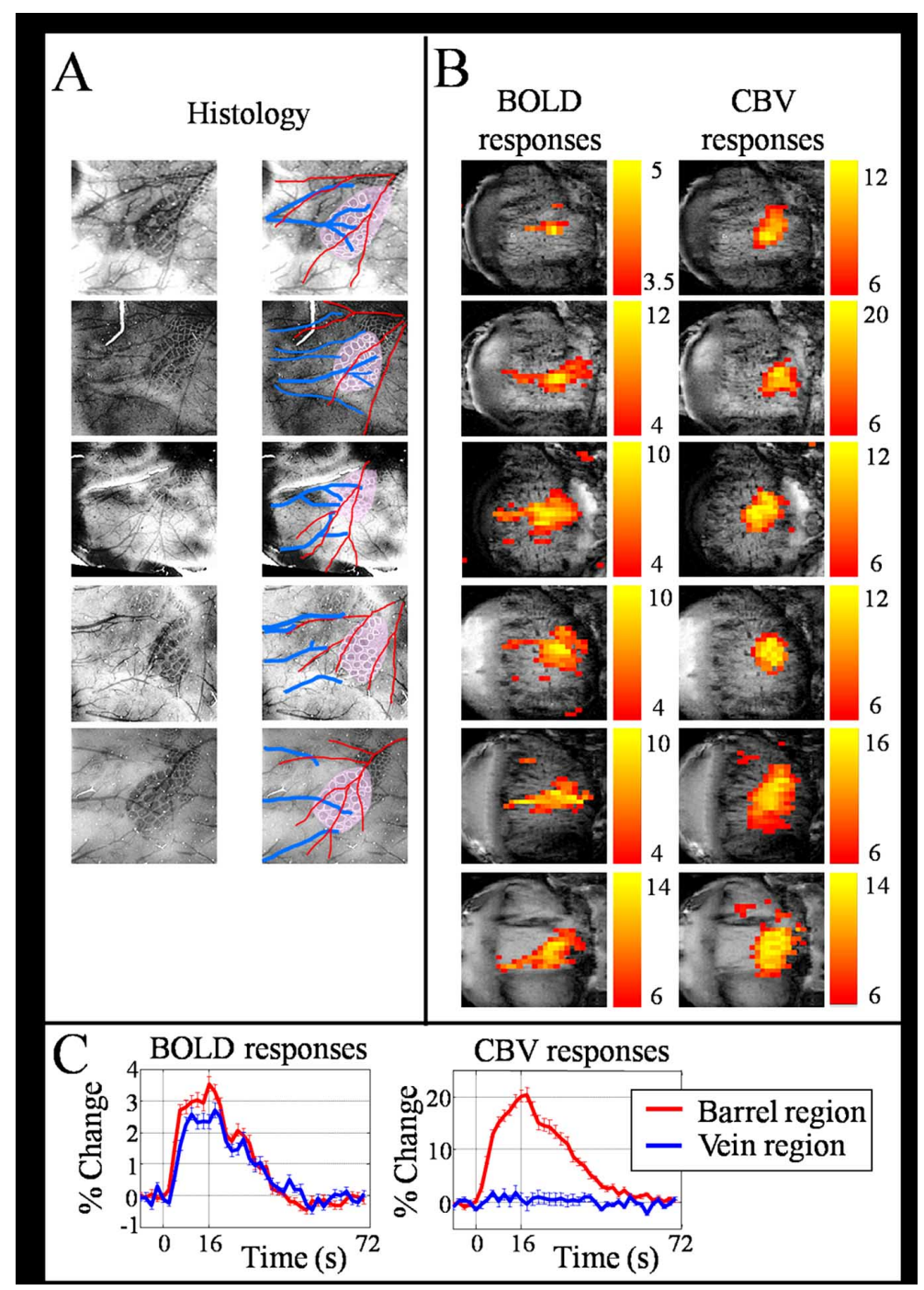

Fig. (2). Direct comparison of imaging fields. We show high correlation between the vascular structure shown in (a) post mortem histological sections and (b) fMRI BOLD and CBV statistical maps for 5 subjects (post-mortem histology was not performed on 1 animal). In particular in all animals we found a significant BOLD response in the large veins running in a lateral-medial direction draining the active area of parenchyma containing the whisker barrel field. No commensurate changes in CBV were observed in the draining veins. Data suggested a high correlation of CBV changes to the barrel region and overlying arterial structures (running in an anterior-posterior direction). Two regions of interest were selected; one covered the barrel cortex and the second was centred on the draining vein region. Averaged times series across trials and animals of both BOLD and CBV changes to whisker stimulation resulted (c).

towards the venous draining system while little or no CBV changes were detected in this region.

The Balloon model [27], was originally proposed to explain the BOLD post-stimulus undershoot as a consequence of the expansion of venous vessels (and increase in $\mathrm{CBV}$ ). Although no undershoot was present in the current data (see below), our failure to detect any reliable change in $\mathrm{CBV}$ in regions corresponding to a major draining veins (Fig. 2) is inconsistent with the Balloon account for this large draining vein. Indeed, close examination of the histological sections, Fig. (2c) reveals that the main area of CBV activity overlapped the barrel cortex and middle cerebral artery (MCA) in an anterior-posterior direction. Data supports the idea that CBV changes are greater in the arterial than the venous vasculature [29] and is in agreement with other recent physiological and MR reports [10, 30-32]. However, the current study cannot discriminate the smaller arterioles and venuoles where the Balloon model may still hold.

Our results exhibited three important temporal aspects that also warrant discussion.

Firstly, our BOLD data failed to show evidence of an initial dip in response to whisker stimulation. It is however possible that recording at the field strength of $3 \mathrm{~T}$ with an effective temporal resolution of $2 \mathrm{~s}$ was insufficiently sensitive to detect initial increases in deoxyhemoglobin (often less that $1 \%$ and lasting less than $1 \mathrm{~s}$ in duration).

Secondly, we found that the BOLD response recorded from the venous compartment lagged that measured in the barrel cortex by $0.55 \mathrm{~s} \pm 0.09$. Delays have been reported previously by others $[23,25]$ and are often attributed to the longer time required for fresh oxygenated blood to reach the larger draining vessels. The mean distance between the 
centres of the two regions in our experiments was $3.15 \mathrm{~mm} \pm$ 0.24 . Therefore, the minimum velocity of blood in this draining vein was estimated to be $6.32 \mathrm{~mm} / \mathrm{s} \pm 0.56$. Although Klienfeld et al. [36] found the flux of red blood cells in the capillaries to be $2 \mathrm{~mm} / \mathrm{s}$, it would be expected that the velocity of blood in the larger draining veins would increase. Supporting this supposition, a cross correlation analysis of optical signals from a similar preparation by Johnston et al. [37] showed that blood velocity was higher in veins $(3-6 \mathrm{~mm} / \mathrm{s})$ than in capillaries $(2 \mathrm{~mm} / \mathrm{s})$.

Thirdly, time series of BOLD signal changes in both the barrel and vein regions do not show a post-stimulus undershoot. The BOLD post stimulus undershoot could be caused by cerebral blood flow returning to baseline faster than blood volume [38], as in the Balloon model. Alternatively, the post stimulus undershoot could be a consequence of reduced cerebral blood flow following stimulation [39, 40]. The sensitivity of our system might account for our failure to observe the post stimulus undershoot. Using a field strength of $11.7 \mathrm{~T}$ Keilholz et al. [6] measured a post stimulus undershoot of approximately $1 \%$. Moreover, their peak BOLD signal was also much greater at $8 \%$ - c.f. $2.5-3.0 \%$ in the present study. Although they stimulated the forepaw, equations of $\Delta R_{2}^{*}$ [4] show that the BOLD signal magnitude is linear with magnetic field strength. Therefore, a BOLD post-stimulus undershoot of around $0.25 \%$ would be predicted at $3 \mathrm{~T}$, which in our equipment would have been masked by system noise. However, it is important to note that in the current study CBV changes in barrel cortex return to baseline almost $16 \mathrm{~s}$ after the BOLD signal (Fig. 2). This is in contrast to Tuunanen et al. [40] who showed that vascular space occupancy (VASO) measurements of CBV changes in humans were no different to basal levels during a recorded BOLD post stimulus undershoot. Although we do not directly measure cerebral blood flow in this present study, our previous work using concurrent fMRI and laser Doppler flowmetry [7] also showed the delayed return to baseline of $\mathrm{CBV}$ changes, and concurrent $\mathrm{CBF}$ changes returning to baseline without an undershoot. Therefore we hypothesise that in the rat the Balloon model is the only reasonable explanation for the post stimulus undershoot.

Although our data challenge the Balloon model as an explanation of the venous BOLD signal in the large draining veins, other explanations of the differences in the venous BOLD and CBV responses should also be considered: i) the imaging method, ii) vascular weighting functions, iii) orientation of the vein in the $\mathrm{B}_{0}$ field, iv) BOLD contamination of the $\mathrm{CBV}$ measurements, or $\mathrm{v}$ ) inflow effects. These possibilities will be discussed below.

\section{i) Imaging Methodology}

In the current study we used a gradient echo - echo planar imaging (GE-EPI) pulse sequence which is sensitive to changes in $\mathrm{R}_{2} *$ caused by field in-homogeneities generated by localised changes in paramagnetic Hbr. Unlike spin echo techniques, the GE-EPI pulse sequence does not have a $180^{\circ}$ refocusing $\mathrm{RF}$ pulse and so is sensitive to oxygenation changes not only in the activated parenchyma but also in and around the large vessels. This is because, as blood vessel radius increases, susceptibility-induced dephasing overshadows diffusion-averaging effects $\left(\mathrm{T}_{2}\right)$. If arterial blood is fully oxygenated and exhibits maximal MR signal intensity [4], then during activation the area of activity will be inherently drawn to the draining venous system. However, the same imaging method was used when we measured CBV changes with contrast agent enhanced MRI. Under these conditions we failed to see a dominant CBV response in the draining veins.

Keilholz et al. [6] imaging in the coronal plane using spin echo techniques also found evidence for a significant BOLD response in the draining venous structure. They showed that the BOLD signal changes were weighted towards the cortical surface where the large vessels reside. High resolution FLASH images indicate the presence of a large vessel in areas of maximum BOLD signal changes. However, the coronal sections are not ideal for identifying whether the vessel is running medial-lateral or anterior-posterior. Both these points are evidence that our imaging methodology was not responsible for amplifying the venous BOLD signal.

\section{ii) Vascular Weighting Functions}

Our observation of different regional sensitivities for BOLD and CBV $f$ MRI is not novel. Coronal maps of BOLD and $\mathrm{CBV}$ have a small shift between activation centroids [14] with BOLD signals originating in the superficial cortex and CBV changes occurring at a depth of approximately $1 \mathrm{~mm}[6,7]$. Using a theoretical analysis of the vascular weighting function, Mandeville \& Marota [14] concluded that CBV $f$ MRI should be less sensitive to the changes in large blood vessels because the vascular weighting function penalises very high blood volume fractions. Alternatively, BOLD biophysics shows a linear monotonic increasing dependence on resting state blood volume which, in the present conditions, would lead to signal amplification in the large arteries and veins. However, if the arterial vasculature compartment was fully oxygenated at the outset it would not be expected to show any BOLD signal changes. If one assumes that the CBV activation maps high-light arterial structures, the difference in spatial distribution between BOLD and CBV could be accounted for by the lack of arterial BOLD signal.

Aligning the maps of CBV changes onto corresponding histological sections (Fig. 2) showed that large increases in CBV had occurred in arterial structures and the surrounding parenchyma with little or no changes in the venous compartment. This is in agreement with Berwick et al. [30] in which optical imaging spectroscopy showed the largest increases in total hemoglobin (associated with CBV) evoked by stimulation of the whisker pad originated predominantly in the arterial vasculature [10, 30-32]. Arteries, like veins, have high blood volume fractions, and thus, if the venous CBV is penalised due to high blood volume fractions then related arteries should similarly be affected. Hence, it is unlikely that the vascular weighting function can account for the absence of venous volume changes.

\section{iii) Angle of the Vein to the $B_{0}$ Field}

The large BOLD signal seen in the draining vein can be partially explained by using the vector model of the BOLD signal [41]. The $f$ MRI signal originates from both the intra (IV) and extra vascular (EV) spaces. As the magnetic field 
strength increases the contribution from the IV space diminishes. At the field strength used in this study (3T) $67 \%$ of the total BOLD signal will have originated from the EV space [42]. By the vector model of the BOLD signal in the EV space the changes in resonant frequency are linearly dependent on the sine squared of the angle between the vessel and the $\mathrm{B}_{0}$ field. In barrel cortex this means little since there are lots of capillaries orientated at many different angles to the $\mathrm{B}_{0}$ field. Consequently, observations from this compartment will have been comparatively independent of angle. However, the histological sections in Fig. (2) show draining veins were consistently orientated in a mediallateral direction. Therefore the draining veins can be regarded as essentially large cylinders lying approximately $90^{\circ}$ to the $\mathrm{B}_{0}$ field. Protons in the EV space around this vessel will therefore experience a greater change in transverse relaxation rate for the same physiological changes. This in turn would generate large BOLD signals in vessels orientated at $90^{\circ}$. However, the same would have been true for our CBV measurement, which relies on changes in relaxation induced by a paramagnetic contrast agent. So, while the vector model offers a good explanation for the large BOLD signal change seen in the draining veins it provides additional support for the argument that these changes occur in the absence of any corresponding alteration of CBV, and are solely an effect of oxygenation.

\section{vi) BOLD Contamination of the CBV Measurements}

The CBV signal measured in the venous region could have been contaminated by the larger observed BOLD signal [43]. During activation the BOLD signal (a decrease in paramagnetism) will be in opposition to the MION contrast enhanced signal (an increase in paramagnetism). Assuming additive relaxation rates during activation the measured relaxation rate is given by:

$\Delta R_{2}^{* M I O N+B O L D}=\Delta R_{2}^{* M I O N}+\Delta R_{2}^{* B O L D}$

Assuming that the relaxation rate due to the MION, $R_{2}{ }^{* M I O N}$, is proportional to local CBV [12], measurements of CBV changes are systematically underestimated, due to the BOLD effect, with a percentage error given by:

$\sigma_{\frac{C B V}{C B V_{0}}}=\frac{\Delta R_{2}^{* B O L D}}{\Delta R_{2}^{* M I O N+B O L D}-\Delta R_{2}^{* B O L D}}$

For the current data set we have a $\sim 7 \%$ error in our measurements of fractional CBV changes in both the parenchyma and vein regions. This systematic error was below the statistical error due to biological variability. Therefore the $10 \mathrm{mg} / \mathrm{kg}$ dose of MION used in the experiments outlined was high enough to minimise BOLD contamination of CBV-weighted $f \mathrm{MRI}$ [11].

\section{v) Inflow Effects}

Studies showing the 'draining vein' often use BOLD functional maps in a coronal anatomical reference frame [6]. It is argued that the effect may therefore be in part caused by an influx of fresh unsaturated spins which will contribute their full magnetisation to scans which have a strong $T_{1}$ weighting of the longitudinal equilibrium magnetisation [44]. This contribution will increase with increased cerebral blood flow and is known as the 'in-flow' effect. Because of the inflow effect, the interpretation of GE-EPI BOLD functional brain maps is sometimes questioned.

Inflow effects are seen as one of the potential pitfalls of functional MRI using conventional gradient echo techniques [45]. If a pulse sequence employs a short TR time and large flip angle, it is said to be $T_{1}$ weighted. Stationary spins in the imaging plane will not relax fully in the longitudinal plane before RF excitation repeats leading to MR signal attenuation. Inflowing spins, which have not previously experienced RF excitation, will contribute their full magnetization. This means that the steady state magnetization of spins is highly dependent upon the rate at which saturated spins are replenished. During a baseline period this effect is irrelevant. However, during neural activity there is a dramatic increase in both blood flow and volume and hence more spins are replenished (with fresh, non-saturated ones) and they will increase the MR signal. This means that MR signal changes could be related to changes in flow rather than oxygenation.

There have been many studies investigating how the inflow effects can be removed from $f$ MRI in several ways. One can apply bipolar 'crushing' gradients [46, 47]. Diffusion weighted studies like this eliminate both inflow effects and the intravascular component of the BOLD signal changes. At the current field strength (3T) the intravascular component of the BOLD signal changes accounts for approximately $1 / 3$ of the total measured changes [48]. In the current study we applied a bi-polar gradient sequence to investigate the inflow effect. We found (see supplementary figure) that the magnitude of the BOLD signal changes in response to electrical stimulation of the whisker pad was reduced by $1 / 3$. As the reduction in signal magnitude was in agreement with a recent study by Lu et al. [42] and Monte Carlo simulations [48] both done at the same magnetic field strength this indicates that only the intravascular component has been removed and therefore no inflow effects are present in the current data. One can further check this conclusion by reducing the $T_{1}$ weighting of the scans. This can be done by either extending the TR (at a cost to temporal resolution) or by altering the flip angle so all spins can relax to maximum longitudinal equilibrium magnetisation [44]. There are also post-acquisition techniques for the suppression of large vessel BOLD signals [41]. Studies in humans have shown that such methods can reduce inflow effects, but not remove them completely [45]. In rats, however, Keilholz et al. [6] found that by extending repetition time from 1 s to 10 s (thus eliminating any $T_{1}$ weighting) the draining vein in somatosensory cortex was still clearly visible in maps of changes in BOLD signal. This indicated that the measured signal was due to changes in blood oxygenation rather than in-flow effects. This result was confirmed in the present study by imaging in the tangential plane which, due to the rat angio-architecture of barrel cortex, minimises in-flow effects.

Imaging in the tangential plane was chosen because there is remarkable spatial arrangement of the blood vessels supplying the barrels in rodent somatosensory cortex [49]. The middle cerebral artery (MCA) runs in an anteriorposterior direction across the surface of the cortex to supply the barrels. Vessels branch in cortical layers I and II and arborize in layers II-IV. In layer $\mathrm{V}$ the vessels spread 
tangentially. Veins on the cortical surface drain the area medially into the superior sagittal sinus (Fig. 1). Our tangential imaging plane encompassed this unique vasculature. The slice thickness was $2 \mathrm{~mm}$ covering all cortical lamina. Unsaturated spins reached equilibrium magnetisation in the arterial tree during both baseline and activation and so did not affect the signal from either the barrel region or draining veins. We considered that this would eliminate potential inflow effects. As our results show there was a clear BOLD signal in the vein region without any corresponding change in $\mathrm{CBV}$. Using 2D optical imaging spectroscopy, which also images the cortical surface, we showed previously that oxygenation changes in the veins were associated with much reduced volume changes when compared to that seen in arteries and the barrel region [30]. This result predicted the significant BOLD signal observed in the venous region using $f$ MRI. Our $f$ MRI results therefore confirm that following activation there is a substantial decrease in deoxyhemoglobin in the major draining vein. Although the opposite result has been reported in human studies the reason for this is unclear at present but could be related to anaesthesia or the stimulation paradigm.

\section{CONCLUSION}

The relative levels of a stimulation evoked activation of BOLD and CBV-weighted $f$ MRI signals differed between vascular compartments. By comparing novel tangential $f$ MRI activation maps to corresponding histological sections a significant BOLD signal change was observed in the draining veins which occurred in the absence of a corresponding $\mathrm{CBV}$ change. In contrast to the draining vein, data can be used to hypothesize that there are significant CBV changes in the arterial supply. This challenges classical explanations of venous BOLD signal and significant venous CBV changes but only in the large draining veins. In regions of brain parenchyma in barrel cortex we have shown substantial changes in both $\mathrm{CBV}$ and BOLD signals in response to sensory stimulation. This compartment contains smaller venuoles which may exhibit CBV changes caused by mechanical expansion both during and post stimulation. Further studies at higher spatial resolution are required before any definitive conclusion in this compartment can be made.

\section{ACKNOWLEDGMENTS}

We gratefully acknowledge support from the National Institutes of Health, Medical Research Council and the Wellcome Trust. We are grateful to Guerbet Pharmaceutical Technologies for providing the MRI contrast agent Sinerem. We thank the laboratory technical staff (Marion Simkins, Natalie Kennerley, Michael Port and Malcolm Benn) for their assistance.

\section{SUPPLEMENTARY MATERIAL}

This article is accompanied by 1 supplementary file which can be viewed at http://www.bentham.org

\section{REFERENCES}

[1] Kwong KK, Belliveau JW, Chesler DA, et al. Dynamic magnetic resonance imaging of human brain activity during primary sensory stimulation. Proc Natl Acad Sci USA 1992; 89(12): 5675-9.
[2] Ogawa S, Lee TM, Kay AR, Tank DW. Brain magnetic resonance imaging with contrast dependent on blood oxygenation. Proc Natl Acad Sci USA 1990; 87(24): 9868-72.

[3] Buxton RB, Frank LR. A model for the coupling between cerebral blood flow and oxygen metabolism during neural stimulation. J Cereb Blood Flow Metab 1997; 17(1): 64-72.

[4] Yablonskiy DA, Haacke EM. Theory of NMR signal behavior in magnetically inhomogeneous tissues: the static dephasing regime. Magn Reson Med 1994; 32(6): 749-63.

[5] Mandeville JB, Marota JJA, Kosofsky B, et al. Dynamic functional imaging of relative cerebral blood volume during rat forepaw stimulation. Magn Reson Med 1998; 39: 615-24.

[6] Keilholz SD, Silva AC, Raman M, Merkle H, Koretsky AP. BOLD and $\mathrm{CBV}$ weighted functional magnetic resonance imaging of rat somatosensory system. Magn Reson Med 2006; 55: 316-24.

[7] Kennerley AJ, Berwick J, Martindale J, Johnston D, Papadakis N, Mayhew J. Concurrent fMRI and optical measures for the investigation of the haemodynamic response function. Magn Reson Med 2005; 54(2): 354-65.

[8] Kida I, Kennan RP, Rothman DL, Behar KL, Hyder F. Highresolution $\mathrm{CMR}(\mathrm{O} 2)$ mapping in rat cortex: a multiparametric approach to calibration of BOLD image contrast at 7 Tesla. J Cereb Blood Flow Metab 2000; 20(5): 847-60.

[9] Kida I, Rothman DL, Hyder F. Dynamics of changes in blood flow, volume, and oxygenation: implications for dynamic functional magnetic resonance imaging calibration. J Cereb Blood Flow Metab 2006; 27(4): 690.

[10] Kim T, Hendrich KS, Masamoto K, Kim SG. Arterial versus total blood volume changes during neural activity-induced cerebral blood flow change: implication for BOLD fMRI. J Cereb Blood Flow Metab 2007; 27(6): 1235-47.

[11] Lu H, Patel S, Luo F, et al. Spatial correlations of laminar BOLD and $\mathrm{CBV}$ responses to rat whisker stimulation with neuronal activity localized by Fos expression. Magn Reson Med 2004; 52(5): 1060-8.

[12] Mandeville JB, Jenkins BG, Chen YC, et al. Exogenous contrast agent improves sensitivity of gradient-echo functional magnetic resonance imaging at 9.4 T. Magn Reson Med 2004; 52(6): 127281 .

[13] Mandeville JB, Jenkins BG, Kosofsky BE, Moskowitz MA, Rosen BR, Marota JJ. Regional sensitivity and coupling of BOLD and CBV changes during stimulation of rat brain. Magn Reson Med 2001; 45(3): 443-7.

[14] Mandeville JB, Marota JJ. Vascular filters of functional MRI: spatial localization using BOLD and CBV contrast. Magn Reson Med 1999; 42(3): 591-8

[15] Silva AC, Koretsky AP, Duyn JH. Functional MRI impulse response for BOLD and CBV contrast in rat somatosensory cortex. Magn Reson Med 2007; 57(6): 1110.

[16] Stefanovic B, Schwindt W, Hoehn M, Silva AC. Functional uncoupling of hemodynamic from neuronal response by inhibition of neuronal nitric oxide synthase. J Cereb Blood Flow Metab 2006; 27(4): 741

[17] Van Camp N, Peeters RR, Van der Linden A. A Comparison between blood oxygenation level-dependent and cerebral blood volume contrast in the rat cerebral and cerebellar somatosensoric cortex during electrical paw stimulation. J Magn Reson Imaging 2005; 22(4): 483

[18] Wu G, Luo F, Li Z, Zhao X, Li SJ. Transient relationships among $\mathrm{BOLD}, \mathrm{CBV}$, and $\mathrm{CBF}$ changes in rat brain as detected by functional MRI. Magn Reson Med 2002; 48(6): 987-93.

[19] Nagaoka $T$, Zhao $F$, Wang $P$, et al. Increases in oxygen consumption without cerebral blood volume change during visual stimulation under hypotension condition. J Cereb Blood Flow Metab 2006; 26(8): 1043.

[20] Yacoub E, Ugurbil K, Harel N. The spatial dependence of the poststimulus undershoot as revealed by high-resolution BOLD-and CBV-weighted fMRI. J Cereb Blood Flow Metab 2006; 26: 63444 .

[21] Zhao F, Jin T, Wang P, Hu X, Kim SG. Sources of phase changes in BOLD and CBV-weighted fMRI. Magn Reson Med 2007; 57(3): 520-27.

[22] Smirnakis SM, Schmid MC, Weber B, Tolias AS, Augath M, Logothetis NK. Spatial specificity of BOLD versus cerebral blood volume fMRI for mapping cortical organization. J Cereb Blood Flow Metab 2007; 27(6): 1248. 
[23] Krings T, Erberich SG, Roessler F, Reul J, Thron A. MR blood oxygenation level-dependent signal differences in parenchymal and large draining vessels: implications for functional MR imaging. Am J Neuroradiol 1999; 20(10): 1907-14.

[24] Lai S, Hopkins EM, Haacke EM, et al. Identification of vascular structures as a major source of signal contrast in high resolution 2D and 3D functional activation imaging of the motor cortex at 1.5T: preliminary results. Magn Reson Med 1993; 30: 387-92.

[25] Lee AT, Glover GH, Meyer CH. Discrimination of large venous vessels in time-course spiral blood-oxygen-level-dependent magnetic-resonance functional neuroimagaing. Magn Reson Med 1995; 33: 745-54.

[26] Gati JS, Menon RS, Ugurbil K, Rutt BK. Experimental determination of the BOLD field strength dependence in vessles and tissue. Magn Reson Med 1997; 38: 296-302.

[27] Buxton RB, Wong EC, Frank LR. Dynamics of blood flow and oxygenation changes during brain activation: the balloon model. Magn Reson Med 1998; 39(6): 855-64.

[28] Mandeville JB, Marota JJ, Ayata C, et al. Evidence of a cerebrovascular postarteriole Windkessel with delayed compliance. J Cereb Blood Flow Metab 1999; 19: 679-89.

[29] Lee SP, Duong TQ, Yang G, Iadecola C, Kim SG. Relative changes of cerebral arterial and venous blood volumes during increased cerebral blood flow: implications for BOLD fMRI. Magn Reson Med 2001; 45(5): 791-800.

[30] Berwick J, Johnston D, Jones M, et al. Neurovascular coupling investigated with two-dimensional optical imaging spectroscopy in rat whisker barrel cortex. Eur J Neurosci 2005; 22(7): 1655-66.

[31] Hillman EM, Devor A, Bouchard MB, et al. Depth-resolved optical imaging and microscopy of vascular compartment dynamics during somatosensory stimulation. NeuroImage 2007; 35(1): 89-104.

[32] Iadecola C, Yang G, Ebner TJ, Chen G. Local and propagated vascular responses evoked by focal synaptic activity in cerebellar cortex. J Neurophysiol 1997; 78(2): 651-9.

[33] Bruder $H$, Fischer $H$, Reinfelder $H$, Schmitt $F$. Image reconstruction for echo planar imaging with noequidistant k-space sampling. Magn Reson Imaging 1992; 23: 311-23.

[34] Friston KJ, Frith CD, Frackowiak RSJ. Comparing functional (PET) images: the assessment of significant change. J Cereb Blood Flow Metab 1991; 11: 690-9.

[35] Zheng Y, Johnston D, Berwick J, Mayhew J. Signal source separation in the analysis of neural activity in brain. Neuroimage 2001; 13(3): 447-58.

[36] Kleinfeld D, Mitra PP, Helmchen F, Denk W. Fluctuations and stimulus-induced changes in blood flow observed in individual capillaries in layers 2 through 4 of rat neocortex. Proc Natl Acad Sci USA 1998; 95(26): 15741-6.

[37] Johnston D, Redgrave P, Berwick J, Kennerley AJ, Mayhew JE. Measurement of cerebral blood flow in rat barrel cortex using optical flow of intrinsic inhomogeniety. San Diego: Conf Soc Neurosci 2007.

[38] Kong Y, Zheng Y, Johnston D, et al. A model of the dynamic relationship between blood flow and volume changes during brain activation. J Cereb Blood Flow Metab 2004; 24(12): 1382-92.

[39] Frahm J, Kruger G, Merboldt KD, Kleinschmidt A. Dynamic uncoupling and recoupling of perfusion and oxidative metabolism during focal brain activation in man. Magn Reson Med 1996; 35(2): $143-8$

[40] Tuunanen PI, Vidyasagar R, Kauppinen RA. Effects of mild hypoxic hypoxia on poststimulus undershoot of blood-oxygenationlevel-dependent fMRI signal in the human visual cortex. Magn Reson Imaging 2006; 24(8): 993-9.

[41] Menon RS. Postacquisition suppression of large-vessel bold signals in high resolution fMRI. Magn Reson Med 2002; 47: 1-9.

[42] Lu H, van Zijl PCM. Experimental measurement of extravascular parenchymal bold effects and tissue oxygen extraction fractions using multi-echo vaso fMRI at $1.5 \mathrm{~T}$ and 3.0T. Magn Reson Med 2005; 53: 808-16.

[43] Harel N, Zhao F, Wang P, Kim SG. Evaluation of the BOLD contribution to the MION fMRI signals. International Conference on Functional Mapping of the Human Brain. NeuroImage 2003; 19(2): S287.

[44] Frahm J, Merboldt KD, Hanicke W, Kleinschmidt A, Boecker H. Brain or vein--oxygenation or flow? On signal physiology in functional MRI of human brain activation. NMR Biomed 1994; 7(1-2): 45-53.

[45] Kim SG, Hendrich K, Hu X, Merkle H, Ugurbil K. Potential pitfalls of functional MRI using conventional gradient-recalled echo techniques. NMR Biomed 1994; 7: 69-74.

[46] Boxerman JL, Bandettini PA, Kwong KK, et al. The intravascular contribution to fMRI signal change: Monte Carlo modeling and diffusion-weighted studies in vivo. Magn Reson Med 1995; 34(1): 4-10.

[47] Zhong J, Kennan RP, Fulbright RK, Gore JC. Quantification of intravascular and extravascular contributions to BOLD effects induced by alteration in oxygenation or intravascular contrast agents. Magn Reson Med 1998; 40(4): 526-36.

[48] Martindale J, Kennerley AJ, Johnston D, Zheng Y, Mayhew JE. Theory and generalisation of Monte Carlo models of the BOLD signal source. Magn Reson Med 2008; 59(3): 607-18.

[49] Patel U. Non-random distribution of blood vessels in the posterior region of the rat somatosensory cortex. Brain Res 1983; 289(1-2): 65-70.

[50] Pawlik G, Rackl A, Bing RJ. Quantitative capillary topography and blood flow in the cerebral cortex of cats: an in vivo microscopic study. Brain Res 1981; 208(1): 35-58.

This is an open access article licensed under the terms of the Creative Commons Attribution Non-Commercial License (http://creativecommons.org/licenses/by-nc/3.0/) which permits unrestricted, non-commercial use, distribution and reproduction in any medium, provided the work is properly cited. 